Francisco Manuel MORENO-CARMONA ${ }^{1}$ Marcelo Fernandes COSTA ${ }^{2}$ Dora Fix VENTURA ${ }^{2}$ Solange Rios SALOMÃO ${ }^{3}$ Paulo Sergio de Moraes BARROS $^{1}$

Correspondência para:

FRANCISCO MANUEL MORENO CARMONA

Laboratório de Oftalmologia Comparada Departamento de Cirurgia

Faculdade de Medicina Veterinária e Zootecnia

Universidade de São Paulo Av. Prof. Dr. Orlando Marques de Paiva, 87 05508-270 São Paulo SP Brasil

fmmoreno@usp.br

Recebido para publicação: 00/00/0000 Aprovado para publicação: 00/00/0000

\title{
Acuidade visual de resolução de grades pelo método dos potenciais visuais evocados de varredura: padronização da metodologia para uso em cães
}

\author{
1 - Laboratório de Oftalmologia Comparada do Departamento de Cirurgia da \\ Faculdade de Medicina Veterinária e Zootecnia da Universidade de São \\ Paulo, São Paulo - SP \\ 2 - Laboratório de Eletrofisiologia Visual e Psicofísica do Departamento de \\ Psicologia Experimental e Núcleo de Neurociências e Comportamento da \\ Universidade de São Paulo, São Paulo - SP \\ 3 - Laboratório de Eletrofisiologia Visual Clínica do Departamento de \\ Oftalmologia da Escola Paulista de Medicina da Universidade Federal de São \\ Paulo, São Paulo - SP
}

\section{Resumo}

O objetivo deste artigo foi avaliar e padronizar a metodologia para medir a acuidade visual (AV) de resolução de grades de cães em vigília pelo método dos potenciais visuais evocados de varredura (PVEV). Foram medidas as acuidades visuais de resolução de grades de 22 cães, 18 filhotes (10 machos, 8 fêmeas) e 4 adultos (2 machos, 2 fêmeas). Os PVEVs (NuDiva) foram registrados a partir de dois eletrodos ativos posicionados no escalpo em $\mathrm{O}_{1}$ e $\mathrm{O}_{2}$, um eletrodo referência posicionado em $\mathrm{O}_{\mathrm{z}}$ e um eletrodo terra em $\mathrm{C}_{\mathrm{z}}$. O estímulo consistiu de padrões reversos de grades de ondas quadradas a uma freqüência de $6 \mathrm{~Hz}$, variando de 0,2 a 12 ciclos por grau (cpg). A luminância média do monitor foi de $159.3 \mathrm{~cd} / \mathrm{m}^{2}$. A distância de teste foi de 50 $\mathrm{cm}$ e o animal permaneceu no colo de seu acompanhante, com a cabeça suavemente contida para manter os olhos voltados ao estímulo do monitor de vídeo (Dotronix D788). O teste foi realizado em sala escura sem o uso de midriáticos, sedativos ou anestésicos. Fomos capazes de medir a acuidade visual em todos os cães. Acompanhamos o desenvolvimento da acuidade visual durante as 13 primeiras semanas de vida quando os filhotes atingiram acuidade semelhante à de adultos. O PVE de varredura é método não invasivo que permite mensurar a AV de forma precisa, sem requerer excessiva cooperação e atenção, do paciente alvo. $\mathrm{O}$ método tem aplicação clínica e investigativa, não sendo necessário o uso de cicloplégicos ou de contenção química.

\section{Introdução}

A avaliação do sistema visual por métodos eletrofisiológicos é empregada na medida de várias funções visuais e, pode auxiliar na localização de eventuais lesões nas vias visuais e na confirmação de doenças oculares $^{1,2}$. Tais métodos visuais foram desenvolvidos para obtenção de evidências da função visual a partir de correlatos eletrofisiológicos, permitindo avaliar a integridade da função retiniana no caso do eletrorretinograma (ERG) ${ }^{3}$ e da via retinogenículo-estriada, no caso do potencial visual evocado (PVE) $)^{2,4,5,6,7,8,9}$.

O ERG é o procedimento eletrofisiológico mais descrito na literatura médica veterinária e é utilizado para diagnosticar doenças retinianas e para a avaliação funcional geral da retina previamente à cirurgia de catarata. O PVE tem recebido menor atenção, embora seja útil para o diagnóstico diferencial entre doenças da retina e de estruturas mais centrais ${ }^{2}$. O PVE 
é registrado como um evento repetitivo extraído do eletroencefalograma (EEG). A promediação do trecho do EEG no qual a resposta evocada ocorre, permite visibilizada, pois elimina as flutuações elétricas irregulares, características do EEG, mantendo apenas os eventos que se repetem.

No PVE de varredura registra-se o EEG durante uma varredura rápida (10s) de estímulos de grades de onda quadrada (ou senoidal) de diferentes larguras (freqüências espaciais), com reversão de padrões em taxa temporal fixa, variando a freqüência espacial continuamente, das menores às maiores freqüências. Assim como o PVE por reversão de padrões, o PVE de Varredura de padrões (PVEV) tem sido utilizado para medida da acuidade visual (AV). Estes testes têm particular importância em pacientes incapazes de participar ativamente dos métodos clínicos do exame oftalmológico e no diagnóstico diferencial entre as doenças retinianas e do nervo óptico, bem como na quantificação da AV. O PVEV é o método que requer menor cooperação do paciente, quando comparado a qualquer outro teste de visão ${ }^{8,9,10}$, o que pode ser uma vantagem em oftalmologia veterinária.

A AV é a capacidade de discriminar os detalhes dos objetos no campo de visão, determinada pela dimensão mínima de alguns aspectos espaciais dos estímulos visuais, referindo-se ao limite espacial da discriminação visual ${ }^{11,12,13,14}$. A AV depende das propriedades ópticas do olho (de gerar uma imagem precisamente focalizada), da capacidade da retina em detectar e processar os estímulos visuais, gerando sinais neurais, e da interpretação desses sinais pelas vias visuais superiores. Considerando os vários estudos sobre AV em cães podemos supor que a AV típica de um cão adulto está por volta de 20/75 $12,13,15,16,17,18,19$. Para efeito de comparação, um indivíduo adulto humano normal tem acuidade visual de 20/20.

Até o momento, um único estudo envolvendo a avaliação da AV de cães pelo PVEV foi relatado em Medicina Veterinária. Três cães adultos, machos, da raça Beagle, avaliados sob sedação e cicloplegia, com idades variando de 1 a 2 anos, foram submetidos a exames de PVEV. Murphy et al. ${ }^{16}$ determinaram a $\mathrm{AV}$ dos animais e o efeito do borramento na mesma, utilizando o método do PVEV, mostrando que mesmo pequenos graus de ametropia causam grande efeito no poder de resolução espacial do animal.

Face à eficácia do PVEV na medida da AV em pacientes não-verbais, objetivouse avaliar a aplicabilidade do método dos PVEVs em cães padronizando a metodologia.

\section{Material e Método}

\section{Animais}

Exames de PVEV foram realizados em 22 cães, sendo 4 cães adultos (Terrier Brasileiro) e 18 filhotes de várias raças (3 Buldogues Ingleses, 1 Husky Siberiano e 14 Terriers Brasileiros). Os filhotes foram examinados a partir da $2^{a}$ ou $3^{a}$ semana de vida, após a abertura da fissura palpebral, e acompanhados até aproximadamente 100 dias de idade.

Foram efetuados exames oftalmológicos freqüentes incluindo avaliação do reflexo pupilar fotomotor (direto e consensual), reflexo de ameaça, teste lacrimal de Schirmer, biomicroscopia em lampada de fenda e oftalmoscopia para garantir a sanidade do sistema visual dos animais.

Este estudo foi aprovado pela Comissão de Bioética da Faculdade de Medicina Veterinária e Zootecnia da Universidade de São Paulo.

\section{Equipamento e Estímulo}

O sistema utilizado para se obter os dados dos PVEs foi o NuDiva (Digital Infant Visual Assesment $)^{20}$. O estímulo consistiu de grades verticais de onda quadrada a uma freqüência de $6 \mathrm{~Hz}$, apresentadas num monitor de vídeo monocromático Dotronix modelo EM 2400 - D 788 com luminância média de $159,5 \mathrm{~cd} / \mathrm{m}^{2}$ e contraste de $80 \%$ constante, compreendendo um ângulo de 
33,6 × 25 graus a $50 \mathrm{~cm}$ de distância. Foi apresentada uma seqüência de 10 freqüências espaciais, da menor à maior durante um intervalo de $10 \mathrm{~s}$, com varredura de freqüência espacial de 0,2 a $12 \mathrm{cpg}$. A taxa temporal de reversão utilizada foi de $6 \mathrm{~Hz}$. Foram obtidas de 3 a 12 repetições da varredura para poder obter uma média de no mínimo três varreduras. As respostas foram obtidas em condição binocular de estimulação a partir de eletrodos de EEG de cúpula de ouro (Grass Gold Disc Electrodes - E6GH). Após a tricotomia da região os eletrodos eram posicionados no escalpo , com creme condutor. Quando necessário, pequenos quadrados de esparadrapo foram utilizados para fixar os eletrodos.

Os PVEVs foram registrados a partir de duas colocações bipolares de eletrodos ativos, $\mathrm{O}_{1}$ e $\mathrm{O}_{2}$, posicionados $2-3 \mathrm{~cm}$ à esquerda e à direita, respectivamente, de um eletrodo de referência comum $(\mathrm{Oz})$, colocado na linha mediana do crânio, na altura das orelhas. O eletrodo terra $(\mathrm{Cz})$ foi colocado $2-3 \mathrm{~cm}$ acima do eletrodo de referência (Figura 1). O posicionamento correto dos eletrodos foi confirmado pelo PVE por flash, realizado em cães adultos.

\section{Procedimento}

Os animais foram posicionados com

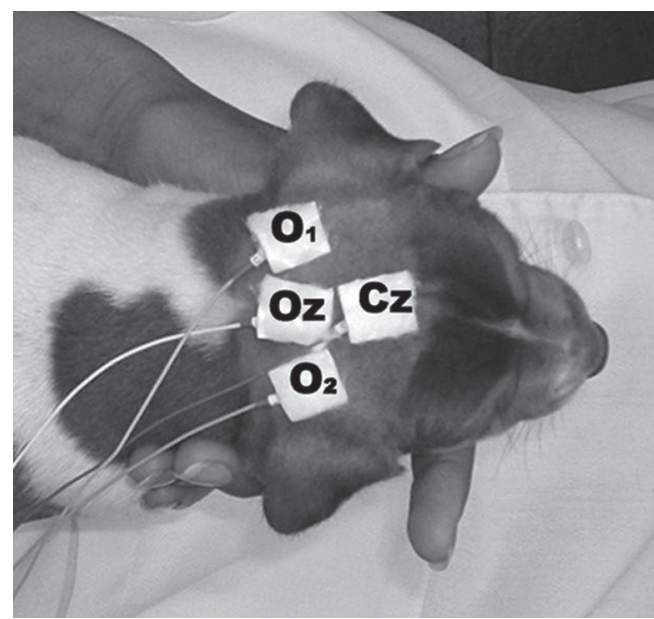

Figura 1 - Posicionamento dos eletrodos ativos $\left(\mathrm{O}_{1} \mathrm{O}_{2}\right)$, do eletrodo comum de referência, $(\mathrm{Oz})$ e do eletrodo terra $(\mathrm{Cz})$, no escalpo tricotomizado do cão em vigília a cabeça levemente contida, à distância de $50 \mathrm{~cm}$ do monitor (Figura 2). Com a sala escura, e com o animal em vigília, quando necessário, a atenção do cão era atraída para a tela do monitor por pequenos brinquedos, apresentados $1-2 \mathrm{~cm}$ em frente à tela durante o período de $10 \mathrm{~s}$ de cada registro. $\mathrm{O}$ experimentador julgava se o animal estava ou não fixando o monitor pelo reflexo do mesmo no centro das pupilas do animal e em caso positivo o EEG era então registrado simultaneamente a partir dos dois canais e filtrado em tempo real para isolar o PVE.

\section{Extrapolação da medida da AV}

Os registros do PVE obtidos eram

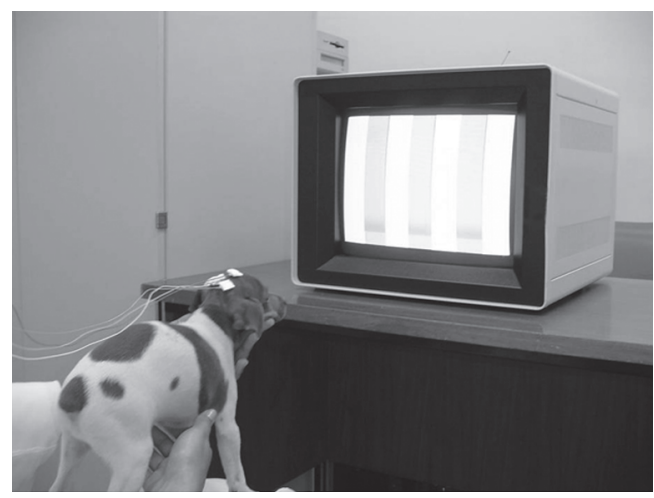

Figura 2 - Posicionamento do animal, no colo de seu acompanhante, a uma distância de $50 \mathrm{~cm}$, à frente do monitor devídeo para a realização do exame de PVE de Varredura. Pode-se observar o padrão de grades de onda quadrada utilizadas como estímulo. Foto apenas ilustrativa: durante a situação de exame os olhos ficavam alinhados como centro do monitor e o exame foi realizado em sala escura

automaticamente analisados pelo aplicativo NuDiva. O aplicativo realiza a transformada discreta de Fourier, extraindo do PVE a amplitude $(\mathrm{mV})$ e a fase (graus) sobre uma faixa de $1 \mathrm{~Hz}$ centralizada em $12 \mathrm{~Hz}$, ou seja, no segundo harmônico da taxa de 6 reversões por segundo. As amplitudes obtidas para cada freqüência espacial são apresentadas em um gráfico no qual um algoritmo automático de extrapolação por regressão linear do pico da amplitude para a amplitude zero era executado para estimar a acuidade visual (Figura 3). Uma relação sinal:ruído de 2:1 foi utilizada como critério 
nas avaliações individuais de registro, enquanto a relação de 3:1 foi utilizada para a obtenção do valor médio final.

Ocorrendo o desvio da direção do olhar por parte do animal ou observandose uma atividade motora importante, como mover as orelhas, o experimentador fazia uma pausa até obter nova fixação ou em caso negativo, o registro era anulado.
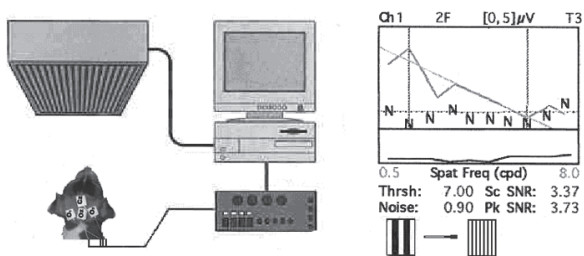

Figura 3 - Esquema ilustrativo da realização do exame de PVEV (esquerda) e da janela do registro, visibilizada pelo experimentador na tela do computador mostrando a curva de resposta obtida (direita). Figura modificada de: < http:// www.ophth.kpu-m.ac.jp/034.htm > Acesso em 28 abr 2004

\section{Resultados}

Com a metodologia adotada para a aplicação do PVEV medimos a AV de resolução de grades de todos os cães, mostrando a alta testabilidade do método empregado. Um registro típico da medida da AV binocular teve a duração de aproximadamente 15 minutos. Os valores de acuidade encontrados foram cofiáveis de acordo com os critérios estabelecidos no

Tabela 1 - Valores individuais da AV de resolução de grades de cão da raça Buldogue Inglês, filhote, avaliado pelo PVEV, no seu primeiromêsdevida:AV (cpg), AV deSnellen-SãoPaulo-2004

\begin{tabular}{ccc}
\hline $\begin{array}{c}\text { IDADE } \\
\text { (semanas) }\end{array}$ & AV (cpg) & $\begin{array}{c}\text { AV } \\
\text { SNELLEN }\end{array}$ \\
\hline 02 & 1,06 & $20 / 565$ \\
03 & 1,82 & $20 / 330$ \\
04 & 3,48 & $20 / 170$ \\
\hline
\end{tabular}

Tabela 2 - Valores individuais da AV de resolução de grades de cão da raça Husky Siberiano, filhote, avaliado pelo PVEV, com 4 , 10 e 15 semanas de vida: AV (cpg), AV de Snellen - São Paulo- 2004

\begin{tabular}{ccc}
\hline $\begin{array}{c}\text { IDADE } \\
\text { (semanas) }\end{array}$ & AV (cpg) & $\begin{array}{c}\text { AV } \\
\text { SNELLEN }\end{array}$ \\
\hline $\mathbf{0 4}$ & 1,17 & $20 / 512$ \\
$\mathbf{1 0}$ & 2,87 & $20 / 200$ \\
$\mathbf{1 5}$ & 9,72 & $20 / 60$ \\
\hline
\end{tabular}

Tabela 3 - Valores individuais da AV de resolução de grades de cão da raça Terrier Brasileiro, filhote, avaliado pelo PVEV, com 4 , 9 e 13 semanas de vida: AV (cpg), AV de Snellen - São Paulo- 2004

\begin{tabular}{ccc}
\hline $\begin{array}{c}\text { IDADE } \\
\text { (semanas) }\end{array}$ & AV (cpg) & $\begin{array}{c}\text { AV } \\
\text { SNELLEN }\end{array}$ \\
\hline 04 & 2,36 & $20 / 255$ \\
09 & 7,53 & $20 / 80$ \\
13 & 8,60 & $20 / 70$ \\
\hline
\end{tabular}

procedimento adotado para todos os animais nas idades investigadas. As tabelas 1, 2 e 3 mostram exemplos de medidas de PVEV realizadas em filhotes de cães. A AV de um dos cães adultos foi de 8,70 cpg (20/ 70). Houve uma progressão continua dos valores de AV nos filhotes testados durante seu desenvolvimento.

\section{Discussão}

A AV dos cães pode ser medida utilizando-se métodos comportamentais ${ }^{12}$, discriminação de formas e aprendizado ${ }^{19}$, nistagmo optocinético ${ }^{15}$, ERG de padrão ${ }^{17,18}$ e $\mathrm{PVE}^{16}$. Entretanto, na prática veterinária clínica, habitualmente, não se mede a AV dos cães com métodos quantitativos ficando, esta avaliação, reservada a medidas qualitativas e 
principalmente pelas informações comportamentais relatadas pelo proprietário dos animais.

Diferentemente dos seres humanos, os cães podem não colaborar de maneira adequada aos testes comportamentais. Na prática pediátrica humana, vem se estudando a capacidade de métodos eletrofisiológicos em medir a função visual, uma vez que tais métodos necessitam de menor colaboração e interação no momento da medida. Estas condições favorecem sua utilização na população canina.

Assim, o PVE tem sido utilizado com relativo sucesso, tanto em seres humanos ${ }^{10,21,22,23}$ quanto em animais sob sedação ${ }^{16,17}$, mais recentemente, visando a predizer os limites da resolução espacial.

De acordo com nossos resultados o PVEV é perfeitamente aplicável na população objeto de nosso estudo. Ao contrário de experimentos com PVE realizados anteriormente nos quais houve a necessidade da contenção química dos animais ${ }^{16,24,25,26}$, nosso estudo evidencia a possibilidade de execução do PVEV em filhotes de cães, bem como em adultos, acordados sem a necessidade de midriáticos, anestesia geral ou tópica nem mesmo de rígida contenção física. A ausência de anestesia melhora a qualidade do registro do teste e o torna clinicamente viável. Este resultado abre novos horizontes na medida em que se torna possível avaliar quantitativamente esta função visual de resolução espacial dos cães. A metodologia utilizada se mostrou capaz de registrar o desenvolvimento da $\mathrm{AV}$.

Encontramos apenas um único relato na literatura do uso de PVEV em cães no qual, Murphy et al. ${ }^{16}$ testaram 03 cães adultos, da raça Beagle, sedados e sob cicloplegia. Tais resultados podem ter sido alterados de alguma forma devido ao uso de midriáticos e sedativos.

A execução do PVEV, em cães, sem o uso de fármacos requer um maior cuidado em sua realização. Algumas vezes o nível de ruído na resposta evocada era grande, em parte, devido ao fato de que nos cães a atividade muscular, bem como a habilidade de movimentar as orelhas, em resposta a estímulos sonoros, serem bastante desenvolvidas. Quando havia algum som externo que chamasse a atenção dos filhotes, a captação da resposta evocada ficava bastante comprometida. O enrugamento da pele entre as orelhas, quando estas eram levantadas, fazia com que os eletrodos saíssem de suas posições originais causando muito ruído. O exame era então momentaneamente suspenso, e quando o filhote retomava a calma o exame era reiniciado. A escolha de mantermos os cães em vigilia para a realização do exame requer alguns cuidados na sua execução, porém em nenhum momento a realização do PVEV ficou comprometida.

Os procedimentos mais comumente utilizados para determinar a visão em cães são rudimentares e qualitativos e baseados em observações clínicas como o teste da sensibilidade da retina a movimentos, a resposta à ameaça ou ainda a habilidade de seguir um objeto qualquer, como por exemplo, um chumaço de algodão em movimento. Porém a confiabilidade de tais testes não é total uma vez que podem ser positivos ainda que a $\mathrm{AV}$ seja muito baixa, por exemplo, 20/400.

O PVEV é eficaz na avaliação quantitativa da $\mathrm{AV}$ em cães, adultos e filhotes sem a necessidade de contenção química, cicloplégicos ou ainda de rígida contenção física nos cães normais. Embora os trabalhos de PVEV existentes sejam, na sua maioria, obtidos para seres humanos ${ }^{10,20,23,27,28,29,30,31}$, esta metodologia apresenta um grande potencial de aplicabilidade em Medicina Veterinária tanto para pesquisa como para a prática clínica. Neste sentido o PVEV vem auxiliar na obtenção de uma medida que condiz com a real capacidade do animal de distinguir detalhes. Quanto maior for o conhecimento que tivermos desta função visual, maior será nossa capacidade de tomar atitudes que culminem numa melhor condição de vida para o animal com deficiência visual.

\section{Agradecimentos}

A FAPESP pela concessão de bolsa (Processo 02/04762-8). 


\section{Grating visual acuity by sweep visually evoked potentials: methodology standardization for use in dogs}

\section{Abstract}

The aim of this article was to evaluate and to standardize the measurement of grating visual acuity in adult and puppy dogs, without any kind of sedation, by sweep visually evoked potentials (sweep-VEP). Grating visual acuities of 22 dogs, 18 puppies (10 males, 8 females) and 4 adults ( 2 males, 2 females) were measured. Sweep-VEPs were recorded from two active electrodes placed on the scalp at $\mathrm{O}_{1}$ and $\mathrm{O}_{2}$, a reference electrode at $\mathrm{O}_{z}$ and a ground electrode at $\mathrm{C}_{\mathrm{z}}$. using as stimulus a pattern reversal square wave grating at a temporal rate of $6 \mathrm{~Hz}$ with spatial frequency, ranging from 0.2 to 12 cycles per degree (cpd). The dog was positioned on the lap of the investigator with the head gently supported to maintain the eyes in front of the video monitor (Dotronix D788), at a distance of $50 \mathrm{~cm}$. The mean luminance was $159.3 \mathrm{~cd} / \mathrm{m}^{2}$. The test was performed in a darkened room without mydriatic, sedative or anesthetic drugs. We were able to obtain grating visual acuity measurements in all dogs. The results were comparable to those obtained with other methods. We also followed the development of grating visual acuity during the first $13^{\text {th }}$ weeks of life when the adult level acuity was reached. The sweep-VEP method is a rapid and reliable procedure for the objective quantification of grating visual acuity in puppies and adult dogs, without the need of sedation.

\section{Referências}

1 BRECELJ, J.; STIRN-KRANJC, B. Eletrophysiologic evaluation of the visual pathway in children - Case reports. Documenta Ophthalmologica, v. 79, p. 313323, 1992.

2 SIMS, M. H. Electrodiagnostic evaluation of vision. In: GELATT, K. N. Veterinary ophthalmology. 3. ed. Baltimore: Lippincott Williams \& Wilkins, 1999. p. 483-507.

3 MARMOR, M. F. et al. Standard for clinical electroretinography (2004 update). Documenta Ophthalmologica, v.108, p. 107-114, 2004.

4 CRUZ, A.; SALOMÃO, S. R. Acuidade Visual. In: DANTAS, A. M.; RODRIGUES, M. L. V. (Org.). Oftalmologia clínica. Rio de Janeiro: Cultura Médica, 2001. v. 1, p. 177-188.

5 KOMAROMY, A. M.; SMITH, P. J.; BROOKS, D. E. Electroretinography in dogs and cats. Part II. Technique, interpretation, and indications. The Compendium on Continuing Education, v. 20, n. 3, p. 355-366, 1998.

6 ODOM, J. V. et al. Visual Evoked Potentials Standard (2004). Documenta Ophthalmologica, v. 108, p. 115123, 2004.

7 OLIVER, J. E.; LORENZ, M. D.; KORNEGAY, J. N. Handbook of veterinary neurology. 3. ed. Philadelphia:
Key-words:

Visual acuity. Electrophysiology. Sweep-VEP. Dogs. Veterinary Ophthalmology.
Saunders, 1997. p. 89-108.

8 OYAMADA, M. K. Eletrofisiologia em neuroftalmologia. In: RODRIGUES-ALVES, C. A. Neuroftalmologia. São Paulo: Roca, 2000. p. 343-362. (Série Atualidades oftalmologia USP; v. III).

9 SALOMÃO, S. R. Eletrofisiologia visual nas uveítes. In: ABREU, M. T. (Org.). Inflamações oculares. São Paulo: Conselho Brasileiro de Oftalmologia, 2002. p. 162-189.

10 COSTA, M. F. et al. Relationship between vision and motor impairment in children with spastic cerebral palsy: new evidence from electrophysiology. Behavioral Brain Research, v. 149, n. 2, p. 145-150, 2004.

11 LOMAS, C. A.; PIGGINS, D.; PHILLIPS, C. J. C. Visual awareness. Applied Animal Behavior Science, v. 57 , p. $247-257,1998$

12 MILLER, P. E.; MURPHY, C. J. Vision in dogs. Journal American Veterinary Medical Association, v. 207, n. 12, p. 1623-1634, 1995.

13 OFRI, R. Optics and physiology of vision. In: GELATT, K. N. Veterinary ophthalmology. 3. ed. Baltimore: Lippincott Williams \& Wilkins, p. 183-216. 1999.

14 WESTHEIMER, G. Adler's physiology of the eye: clinical application. 9. ed. St. Louis: William Hart Jr. 1992. p. 531-546. 
15 EZEH, P. I. et al. Utilizing an optokinetic device in assessing the functional visual acuity of the dog. Progress in Veterinary Neurology, v. 1, n. 4, p. 427-432, 1990.

16 MURPHY, C. J. et al. Effect of optical defocus on visual acuity in dogs. American Journal of Veterinary Research, v. 58, n. 4, p. 414-418, 1997.

17 ODOM, J. V.; BROMBERG, N. M.; DAWSON, W. W. Canine visual acuity: retinal and cortical field potentials evoked by pattern stimulation. American Journal of Physiology, v. 245, p. 637-641, 1983.

18 OFRI, R.; DAWSON, W. W.; GELATT, K. N. Visual resolution in normal and glaucomatous dogs determined by pattern electroretinogram. Progress in Veterinary and Comparative Ophthalmology, v. 3, p. 111-116, 1993.

19 TANAKA, T. et al. Studies on the visual acuity of dogs using shape discrimination learning. Animal Science Journal, v. 71, n. 6, p. 614-620, 2000.

20 NORCIA, A. M.; TYLER, C. W. Spatial frequency sweep vep: visual acuity during the first year of life. Vision Research, v. 25, n. 10, p. 1399-1408, 1985.

21 DOBSON, V.; TELLER, D. Y. Visual acuity in human infants: a review and comparison of behavioral and electrophysiological studies. Vision Research, v. 18, p. 1469-1483, 1978.

22 MAFFEI, L.; FIORENTINI, A. The pattern electroretinogram in animals and humans: physiological and clinical. In: COHEN, B.; BODIS WOLLNER, I. (Ed.). Vision and the brain. New York: Raven Press, 1990. p. 289-296.

23 SACAI, P. Y. et al. Contribuição diagnóstica da avaliação eletrofisiológica visual em pacientes atendidos em hospital universitário. Arquivos Brasileiros de Oftalmologia, v. 66, p. 177-181, 2003.

24 HOWARD, D. R.; BREAZILE, J. E. Normal visual cortical-evoked response in the dog. American Journal of Veterinary Research, v. 33, n. 11, p. 2155-2157, 1972.

25 MALNATI, G. A.; MARSHALL, A. E.; COULTER, D. $B$. Electroretinographic components of the canine visual evoked response. American Journal of Veterinary Research, v. 42, p. 159-163, 1981.

26 SIMS, M. H. et al. Waveform analysis and reproducibility of visual-evoked potentials in dogs. American Journal of Veterinary Research, v. 50, n. 11, p. 1823-1828, 1989.

27 GOOD, W. V. Development of a quantitative method to measure vision in children with chronic cortical visual impairment. Transactions of the American Ophthalmological Society, v. 99, p. 253269, 2001.

28 GOTTLOB, I. et al. Visual acuity measurements by sweep spatial frequency visual-evoked-cortical potentials
(VECPs): clinical application in children with various visual disorders. Journal of Pediatric Ophthalmology \& Strabismus, v. 27, n. 1, p. 40-47, 1990.

29 HARO, F. B. et al. Visual acuity in preterm infants with and without associated risk factors. ARVO abstracts. Investigative Ophthalmology and Visual Science, v. 41, n. 4, p. S624, 2000.

30 OLIVEIRA, A. G. F. et al. Contrast sensitivity threshold measured by sweep-visual evoked potential in term and premature infants at 3 and 10 months of age. Brazilian Journal of Medical and Biological Research, 2004, v. 37, p. 1389-1396, 2004.

31 SOKOL, S. Measurement of infant visual acuity from pattern reversal evoked potentials. Vision Research, $\mathrm{v}$. 18, p. 33-39, 1978.

32 STRAIN, G. M.; OLCOTT, B. M.; HOKETT, L. D. Electroretinogram and visual-evoked potential measurements in Holstein cows. American Journal of Veterinary Research, v. 47, n. 5, p. 1079-1081, 1986. 